\title{
Drug-induced sensorineural deafness caused by antithyroid drugs: a rare side effect
}

\author{
${ }^{1}$ UY Raja, ${ }^{2} \mathrm{~A}$ Kumar, ${ }^{3} \mathrm{~V}$ Possamai, ${ }^{4} \mathrm{D}$ Warner, ${ }^{5} \mathrm{D}$ Barton \\ ${ }^{1}$ Specialty Trainee 5, Diabetes and Endocrinology; ${ }^{2}$ Fixed-term Specialty Trainee, Diabetes and Endocrinology; ${ }^{3}$ Specialist Registrar, Ear, Nose, \\ Throat; ${ }^{4,5}$ Consultant, Diabetes and Endocrinology, Princess Royal Hospital, Telford, UK
}

\begin{abstract}
Acute ototoxicity is a rare but important complication of antithyroid drugs. Although previous cases have been reported in the medical literature, these cases occurred in younger patients with serological evidence of lupus-like syndrome with positive antidouble-stranded deoxyribonucleic acid and antineutrophil cytoplasmic antibodies. We describe the case of a 68-year-old Caucasian male who developed deafness and tinnitus one week after being prescribed carbimazole. The management options include stopping the culprit drug, treatment with immunosuppressive drugs and referring the patient for urgent thyroidectomy. Healthcare professionals who prescribe antithyroid drugs should be aware of this rare but potentially serious complication, so that early drug withdrawal and referral for surgery can be considered.
\end{abstract}

KEYWORDS Carbimazole, deafness, hyperthyroidism

DECLARATION OF INTERESTS No conflict of interests declared.
Correspondence to UY Raja, Diabetes and Endocrinology Department, Birmingham Heartlands Hospital, Bordesley Green East, Birmingham B9 5SS, UK

\section{tel. $+44(0)$ I 2 I 4242000}

e-mail umar_yousaf@hotmail.com

\section{CASE REPORT}

Hyperthyroidism is a common disease with an incidence of $80 / 100,000$ per year in women and $8 / 100,000$ per year in men.' In the UK, it is most usually treated with carbimazole. We report a rare complication of carbimazole-induced deafness in a 68-year-old man.

The patient was referred to our endocrine clinic with symptoms of weight loss, palpitations and hyperkinesia. Hyperthyroidism was confirmed by his thyroid function tests: free T4 $30.1 \mathrm{pmol} / \mathrm{l}$, free T3 $9.3 \mathrm{pmol} / \mathrm{l}$ and thyroidstimulating hormone $<0.05 \mathrm{mU} / \mathrm{l}$. He was prescribed carbimazole, $20 \mathrm{mg}$ daily. A week later the patient developed sudden onset dizziness, together with deafness and tinnitus in his left ear. Similar symptoms developed in his right ear shortly thereafter. His general practitioner diagnosed a 'viral illness'.

Seven weeks after starting carbimazole, the patient attended the ear, nose and throat (ENT) outpatient clinic with severe vertigo, tinnitus and bilateral deafness. He denied otorrhoea or earache. Otoscopy revealed normal tympanic membranes and middle ears bilaterally. A cranial nerve examination was normal, with the exception of the eighth nerve. On tuning-fork testing the patient was unable to hear a Weber test at all, and just able to hear by air conduction, but not bone conduction, in Rinne's test, making this positive bilaterally. Tympanograms revealed normal middle ear pressure. Pure tone audiometry revealed moderate to severe bilateral asymmetrical sensorineural hearing loss (Figure I). Carbimazole was felt likely to be a factor in the development of his symptoms, and his medication was therefore changed to propylthiouracil, $200 \mathrm{mg}$ daily. An

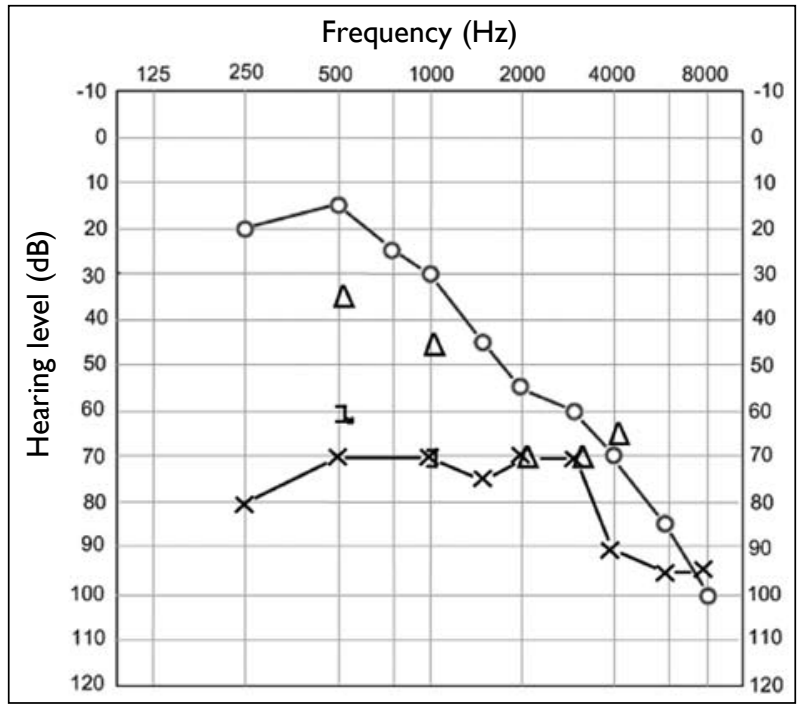

FIGURE I Pure tone audiogram at presentation in the ear, nose and throat clinic showing the patient's severe left sensorineural hearing loss and sloping loss on the right, with preservation of hearing at low tones and increasing loss at higher frequencies. (Circles $=$ air conduction right ear; crosses $=$ air conduction left ear; triangles $=$ unmasked bone conduction right ear; half square $=$ masked bone conduction left ear.)

autoimmune profile, including antinuclear antibody (ANA), antineutrophil cytoplasmic antibodies (ANCA), antidouble-stranded (DS) deoxyribonucleic acid (DNA) antibody and rheumatoid factor, was requested, together with a magnetic resonance imaging scan of the internal acoustic meatus and brain, which excluded central causes of deafness and tinnitus such as tumours or stroke.

On review two weeks later, the patient felt his dizziness was improved, although he remained unsteady on his 
feet. His profound deafness and bilateral tinnitus continued. His audiogram showed a further deterioration on the left side of 5-10 dB across the frequencies. His autoimmune screen was normal, except for a positive extractable nuclear antigen screen with anti-Ro pattern. The patient was started empirically on a three-week trial of prednisolone, $40 \mathrm{mg}$ daily, as some previous case reports demonstrated a beneficial effect of immunosuppressive drugs on improvement of deafness induced by antithyroid drugs. ${ }^{46}$

Following endocrine review, the patient's propylthiouracil was withdrawn in case this was also implicated in his symptomatology. His hyperthyroidism recurred soon after, and he was referred for total thyroidectomy with potassium iodide cover. On subsequent ENT review, the patient's dizziness had improved, although his tinnitus persisted. His hearing continued to deteriorate, particularly in the low tones of the right ear (Figure 2). In view of a lack of response, his prednisolone was withdrawn. The patient underwent total thyroidectomy successfully with a resolution of hyperthyroidism, although his tinnitus and deafness still persist with only slight improvement.

\section{DISCUSSION}

Acute ototoxicity is a rare but important complication of thiourea-derived antithyroid drugs. Although previous cases have been reported in the medical literature, they occurred in younger patients with serological evidence of lupus-like syndrome with anti-DS DNA antibodies ${ }^{2,3}$ and ANCA. ${ }^{4-6}$ There was also a history of an acute illness with fever and joint pains concurrent with the symptoms of deafness and tinnitus. These case reports also suggested an incomplete recovery of deafness after drug withdrawal. Our patient is of interest because of the absence of a history of acute illness; he was also older than the previously reported patient group. His anti-DS DNA and ANCA antibodies were normal with positive anti-Ro antibodies and thus might represent a different mechanism for the development of ototoxicity. Moreover, in our patient the temporal relationship between prescribing carbimazole and the development of deafness, as well as bilateral involvement, suggests carbimazoleinduced deafness rather than idiopathic deafness as his cause of deafness.

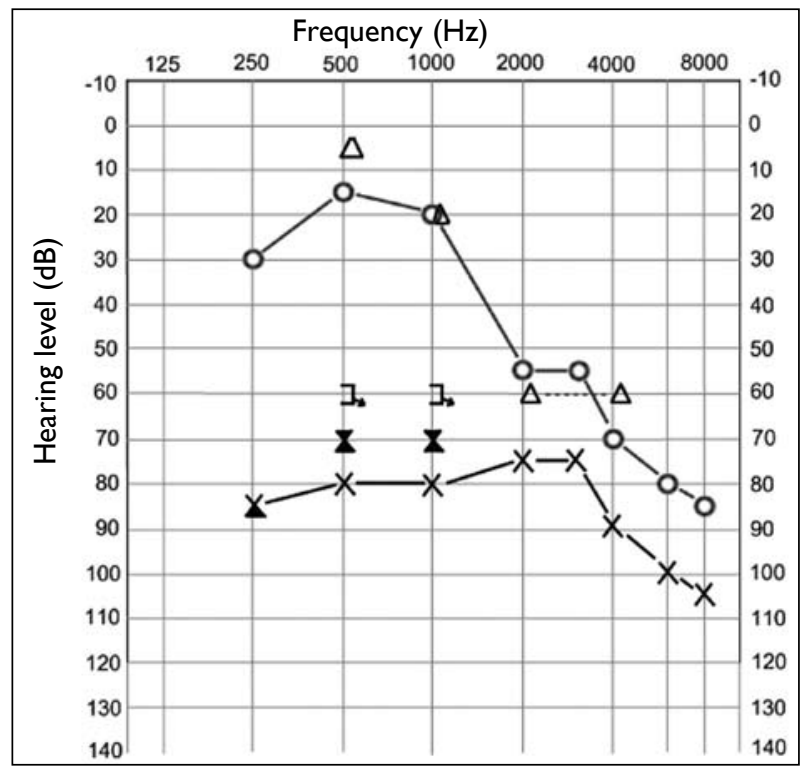

FIGURE 2 Pure tone audiogram following drug withdrawal. In comparison to Figure I there is slight progression of left sensorineural hearing loss. Hearing on the right is essentially unchanged. (Circles $=$ air conduction right ear; crosses $=$ air conduction left ear; triangles = unmasked bone conduction right ear; half square $=$ masked bone conduction left ear.)

The mechanism behind antithyroid drug-associated sensorineural deafness is unknown. It may represent a hypersensitivity reaction involving the inner ear, with the development of autoantibodies against connective tissue or neural antigens in the cochlea. ${ }^{2.5}$ Inner ear blood flow impairment due to antibody-associated small vessel vasculitis may also be a factor. ${ }^{6}$ There seems to be a stronger association between propylthiouracil and autoimmune inner ear disease than with carbimazole therapy. ${ }^{2}$ There is no consensus on definite management, but options include stopping the culprit drug, treatment with immunosuppressive drugs and referring the patient for urgent thyroidectomy. ${ }^{46}$

\section{CONCLUSION}

Healthcare professionals who prescribe antithyroid drugs should be aware of this rare but potentially serious complication so that early drug withdrawal and referral for surgery can be considered.

\section{REFERENCES}

I McGrogen A, Seaman HE, Wright JW et al. The incidence of autoimmune thyroid disease: a systemic review of the literature. Clin Endocrinol 2008; 69:687-96. doi:I0. I I I I/j. I365-2265.2008.03338.x

2 Hill D, Whittet $\mathrm{H}$, Simpson $\mathrm{H}$. Hearing loss and tinnitus with carbimazole. BMJ 1994; 309:929.

3 Fong PC, Pun KK, Tai YT et al. Propylthiouracil hypersensitivity with circumstantial evidence for drug-induced reversible sensorineural deafness: a case report. Horm Res 1991; 35:132-6. doi:10.1159/000181888
4 Thamprajamchit S, Jariengprasert C, Rajatanavin R. Propylthiouracilinduced sensorineural hearing loss associated with antineutrophil cytoplasmic antibodies. Endocr Pract 2004; 10:432-7.

5 Sano M, Kitahara N, Kunikata R. Progressive bilateral sensorineural hearing loss induced by an antithyroid drug. Orl J Otorhinolaryngol Relat Spec 2004; 66:28I-5. doi:I0.1 I59/00008II26

6 Maguchi S, Fukuda S, Chida E et al. Myeloperoxidase-antineutrophil cytoplasmic antibody-associated sensorineural hearing loss. Auris Nasus Larynx 200I; 28:SI03-6. doi:I0.10I6/S0385-8I46(00)00I03-6 